\title{
Commentary: The absence of protein Y4yS affects negatively the abundance of T3SS Mesorhizobium loti secretin, RhcC2, in bacterial membranes
}

\author{
Anastasia D. Gazi * \\ Laboratoire d'Enzymologie et Biochimie Structurales, Centre National de la Recherche Scientifique, Gif-sur-Yvette, France
}

Keywords: secretin, pilotin, rhizobia, type III secretion system, type II secretion system, tight adherence secreton

\section{A commentary on}

The absence of protein Y4yS affects negatively the abundance of T3SS Mesorhizobium loti secretin, RhcC2, in bacterial membranes

\section{OPEN ACCESS}

Edited by:

Mary C. Wildermuth,

University of California, Berkeley, USA

Reviewed by:

Marie-Anne Barny,

Institut National de la Recherche

Agronomique, France

Konstantin V. Korotkov,

University of Kentucky, USA

*Correspondence:

Anastasia D. Gazi,

anadegaz@gmail.com

Specialty section:

This article was submitted to

Plant Biotic Interactions,

a section of the journal

Frontiers in Microbiology

Received: 19 March 2015

Accepted: 29 June 2015

Published: 14 July 2015

Citation:

Gazi AD (2015) Commentary: The absence of protein Y4yS affects

negatively the abundance of T3SS

Mesorhizobium loti secretin, RhcC2, in bacterial membranes.

Front. Microbiol. 6:710.

doi: 10.3389/fmicb.2015.00710 by Mercante, V., Duarte, C. M., Sánchez, C. M., Zalguizuri, A., Caetano-Anollés, G., and Lepek, V. C. (2015). Front. Plant Sci. 6:12. doi: 10.3389/fpls.2015.00012

Bacteria secrete macromolecules to manipulate their surrounding environment (abiotic or biotic one) for their own benefit. Among them, the diderms (Gram-negative), face the challenge of protein transport across an additional, important barrier: the outer membrane (OM). Specific pathways have evolved to transfer lipidated polypeptide chains to the OM, for example, the Lol system (Okuda and Tokuda, 2011). These polypeptides are then inserted to the inner OM leaflet via their $\mathrm{N}$-terminal-attached lipid moiety. Some of them adopt a $\beta$-barrel fold or polymerize in a specific way to accomplish their complete insertion in the membrane. A large family of protein domains, named secretins (Figure 1; Korotkov et al., 2011), that polymerize to form a channel in the OM, is present in many bacterial secretion systems, including the Type II Secretion System (T2SS), the Type IV pili (both T4aP and T4bP types) and the Type III Secretion System (T3SS).

In a generalized scheme, the nascent polypeptide of a secretin carries an $\mathrm{N}$-terminal signal that is recognized by the general secretion pathway (Sec). The Sec system transfers the polypeptide to the periplasmic space. Once there, most often an escorting lipoprotein, pilotin, or docking protein that is also targeted to the periplasmic space via the Sec or Tat pathways will assist with delivery, insertion and/or right polymerization of the polypeptide in the OM, most likely through enrollment of the Lol system (Collin et al., 2011; Okuda and Tokuda, 2011).

Pilotins are extremely diverse in their folding and way of action, while they are usually specific to their cognate secretins (Figure 1; Koo et al., 2012; Dunstan et al., 2013). In most cases, they are encoded within gene clusters that also encode their cognate secretins, as well as other subunits of the respective bacterial secretion system they serve.

Type III secretion systems evolved for the direct translocation of bacterial proteins from the bacterial cytoplasm to the eukaryotic cell cytoplasm (Soto et al., 2006; Tampakaki et al., 2010; Marteyn et al., 2012; Tampakaki, 2014), a process involved in either pathogenesis or symbiosis with higher organisms. These systems appear to have evolved from the bacterial flagellum through gene loss and new gene acquisitions. Several modern T3SS families exist today that are specialized in the invasion of a broad range of hosts, animals or plants (Abby and Rocha, 2012). 


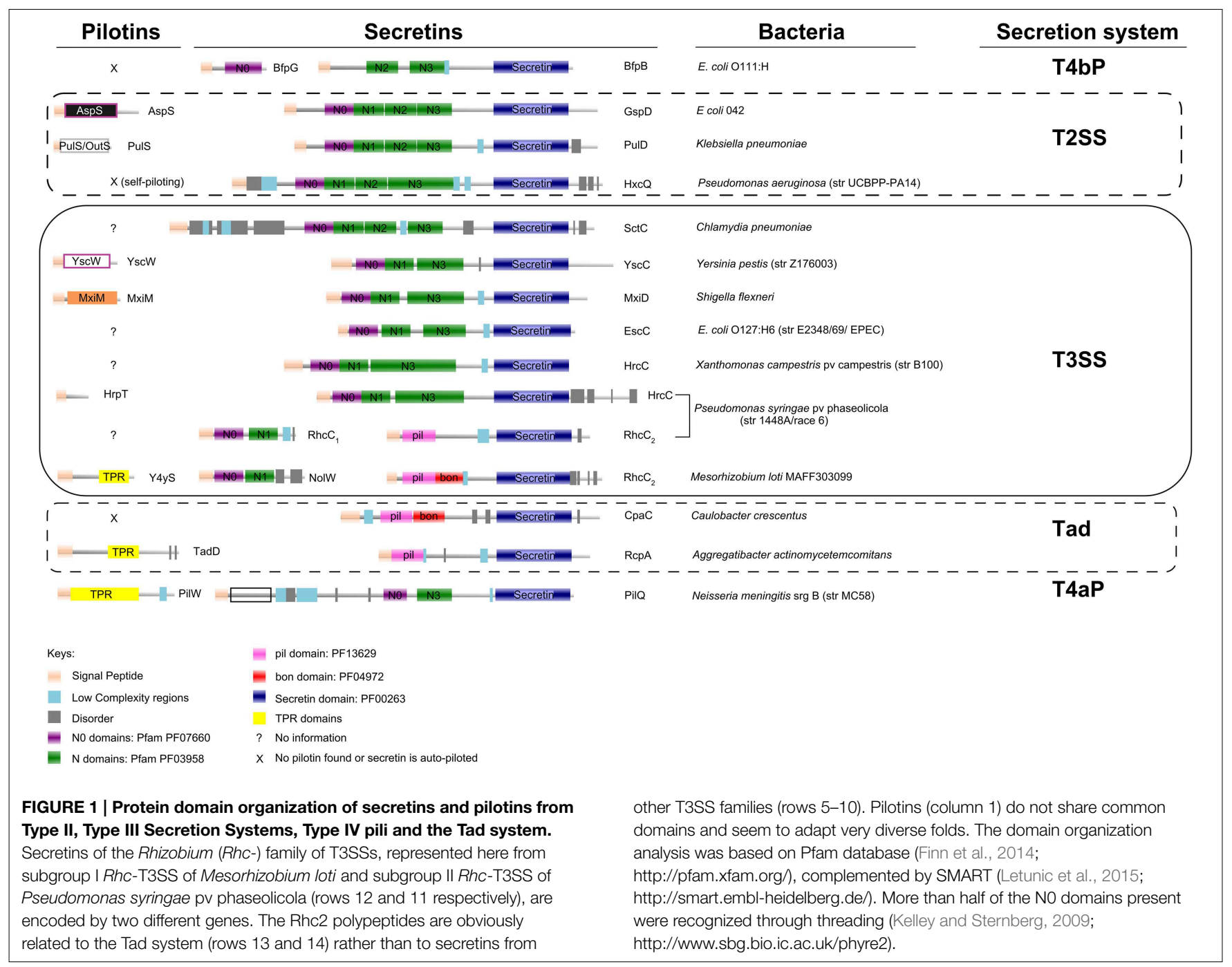

The bacterial flagellum does not possess a secretin while a T2SS/T4P originated secretin has been early acquired and evolved to a specialized T3SS secretin. However, in the case of the T3SS of Rhizobia (Rhc-T3SS), dedicated to symbiosis, this early acquired T2SS/T4P related secretin was lost and only a shorter aminoterminal remnant of this gene (named $r h c C 1$ ), is present in the T3SS gene cluster (Figure 1), while an acquisition of a Tad (Tight Adherence)-like secretin (named $r h c C 2$ ) took place, possibly through DNA recombination events (Figure 1; Abby and Rocha, 2012).

The work of Mercante et al. (2015) nicely complements these findings by showing that a predicted lipoprotein $(y 4 y S)$ of the Mesorhizobium loti Rhc-T3SS could act as a potential pilotin by influencing the level of $\mathrm{RhcC} 2$ protein present in bacterial membranes, while sharing common characteristics (tetatricopeptide repeats, TPRs) of the Tad system pilotins.
Surprisingly, TPR domains have not been observed in other known pilotins within the remaining T3SS families, while they are present in Tad and T4aP pilotins (Figure 1). This new finding, raised new questions. The mechanism by which $\mathrm{Y} 4 \mathrm{yS}$ is piloting the $R h c$-secretin to the OM still remains unknown. Furthermore, $y 4 y S$ homologs are only present in a subset of Rhc-T3SSs (Gazi et al., 2012). Rhc-T3SS subgroup II clusters do not harbor a $y 4 y S$ gene, while Rhc-T3SS subgroup III of Rhizobium etli although maintaining a $r h c C 1$ gene, seems to have lost or never acquired a Tad like secretin $(r h c C 2)$, adding even more mystery to how this particular T3SS nanomachine transverses the OM. Why is a system-specific, non-conserved pilotin needed for the delivery and accommodation of a highly conserved domain in the outer bacterial membrane? This question cannot be answered as of this time, but it seems quite possible that more studies will further reveal this complexity before giving us some first clues. 


\section{References}

Abby, S. S., and Rocha, E. P. C. (2012). The non-flagellar type III secretion system evolved from the bacterial flagellum and diversified into host-cell adapted systems. PLoS Genet. 8:e1002983. doi: 10.1371/journal.pgen.10 02983

Collin, S., Guilvout, I., Nickerson, N. N., and Pugsley, A. P. (2011). Sorting of an integral outer membrane protein via the lipoprotein-specific Lol pathway and a dedicated lipoprotein pilotin. Mol. Microbiol. 80, 655-665. doi: 10.1111/j.13652958.2011.07596.x

Dunstan, R. A., Heinz, E., Wijeyewickrema, L. C., Pike, R. N., Purcell, A. W., Evans, T. J., et al. (2013). Assembly of the Type II secretion system such as found in Vibrio cholerae depends on the novel pilotin AspS. PLoS Pathog. 9:e1003117. doi: 10.1371/journal.ppat.1003117

Finn, R. D., Bateman, A., Clements, J., Coggill, P., Eberhardt, R. Y., Eddy, S. R., et al. (2014). Pfam: the protein families database. Nucleic Acids Res. 42, D222-D230. doi: $10.1093 /$ nar/gkt1223

Gazi, A. D., Sarris, P. F., Fadouloglou, V. E., Charova, S. N., Mathioudakis, N., Panopoulos, N. J., et al. (2012). Phylogenetic analysis of a gene cluster encoding an additional, rhizobial-like type III secretion system that is narrowly distributed among Pseudomonas syringae strains. BMC Microbiol. 12:188. doi: 10.1186/1471-2180-12-188

Kelley, L. A., and Sternberg, M. J. E. (2009). Protein structure prediction on the Web: a case study using the Phyre server. Nat. Protoc. 4, $363-371$. doi: 10.1038/nprot.2009.2

Koo, J., Burrows, L. L., and Howell, P. L. (2012). Decoding the roles of pilotins and accessory proteins in secretin escort services. FEMS Microbiol. Lett. 328, 1-12. doi: 10.1111/j.1574-6968.2011.02464.x

Korotkov, K. V., Gonen, T., and Hol, W. G. J. (2011). Secretins: dynamic channels for protein transport across membranes. Trends Biochem. Sci. 36, 433-443. doi: 10.1016/j.tibs.2011.04.002
Letunic, I., Doerks, T., and Bork, P. (2015). SMART: recent updates, new developments and status in 2015. Nucleic Acids Res. 43, D257-D260. doi: 10.1093/nar/gku949

Marteyn, B. S., Gazi, A. D., and Sansonetti, P. J. (2012). Shigella A model of virulence regulation in vivo. Gut Microbes 3, 104-120. doi: 10.4161/gmic.19325

Mercante, V., Duarte, C. M., Sánchez, C. M., Zalguizuri, A., Caetano-Anollés, G., and Lepek, V. C. (2015). The absence of protein Y4yS affects negatively the abundance of T3SS Mesorhizobium loti secretin, RhcC2, in bacterial membranes. Front. Plant Sci. 6:12. doi: 10.3389/fpls.2015.00012

Okuda, S., and Tokuda, H. (2011). Lipoprotein sorting in bacteria. Annu. Rev. Microbiol. 65, 239-259. doi: 10.1146/annurev-micro-090110-102859

Soto, M. J., Sanjuán, J., and Olivares, J. (2006). Rhizobia and plant-pathogenic bacteria: common infection weapons. Microbiology 152, 3167-3174. doi: 10.1099/mic.0.29112-0

Tampakaki, A. P. (2014). Commonalities and differences of T3SSs in rhizobia and plant pathogenic bacteria. Front. Plant Sci. 5:114. doi: 10.3389/fpls.2014.00114

Tampakaki, A. P., Skandalis, N., Gazi, A. D., Bastaki, M. N., Sarris, P. F., Charova, S. N., et al. (2010). Playing the "Harp": evolution of our understanding of hrp/hrc genes. Annu. Rev. Phytopathol. 48, 347-370. doi: 10.1146/annurevphyto-073009-114407

Conflict of Interest Statement: The author declares that the research was conducted in the absence of any commercial or financial relationships that could be construed as a potential conflict of interest.

Copyright (C) 2015 Gazi. This is an open-access article distributed under the terms of the Creative Commons Attribution License (CC BY). The use, distribution or reproduction in other forums is permitted, provided the original author(s) or licensor are credited and that the original publication in this journal is cited, in accordance with accepted academic practice. No use, distribution or reproduction is permitted which does not comply with these terms. 